\title{
Drug resistance-conferring mutations in Mycobacterium tuberculosis from Madang, Papua New Guinea
}

\author{
Marie Ballif ${ }^{1,2}$, Paul Harino ${ }^{3}$, Serej Ley ${ }^{1,2,3}$, Mireia Coscolla ${ }^{1,2}$, Stefan Niemann ${ }^{4}$, Robyn Carter ${ }^{5}$, Christopher Coulter ${ }^{5}$,
} Sonia Borrell ${ }^{1,2}$, Peter Siba ${ }^{3}$, Suparat Phuanukoonnon ${ }^{3}$, Sebastien Gagneux ${ }^{1,2}$ and Hans-Peter Beck ${ }^{1,2^{*}}$

\begin{abstract}
Background: Monitoring drug resistance in Mycobacterium tuberculosis is essential to curb the spread of tuberculosis (TB). Unfortunately, drug susceptibility testing is currently not available in Papua New Guinea (PNG) and that impairs TB control in this country. We report for the first time $M$. tuberculosis mutations associated with resistance to first and second-line anti-TB drugs in Madang, PNG. A molecular cluster analysis was performed to identify M. tuberculosis transmission in that region.

Results: Phenotypic drug susceptibility tests showed $15.7 \%$ resistance to at least one drug and $5.2 \%$ multidrug resistant (MDR) TB. Rifampicin resistant strains had the rpoB mutations D516F, D516Y or S531L; Isoniazid resistant strains had the mutations katG S315T or inhA promoter C15T; Streptomycin resistant strains had the mutations rpsL K43R, K88Q, K88R), rrs A514C or gidB V77G. The molecular cluster analysis indicated evidence for transmission of resistant strain.

Conclusions: We observed a substantial rate of MDR-TB in the Madang area of PNG associated with mutations in specific genes. A close monitoring of drug resistance is therefore urgently required, particularly in the presence of drug-resistant M. tuberculosis transmission. In the absence of phenotypic drug susceptibility testing in PNG, molecular assays for drug resistance monitoring would be of advantage.
\end{abstract}

Keywords: Mycobacterium tuberculosis, Papua New Guinea, Drug resistance, Mutations

\section{Background}

Mycobacterium tuberculosis drug resistance is a global concern. In Papua New Guinea (PNG), the estimated tuberculosis (TB) incidence rate is $303 / 100000$ population, with 5\% multidrug resistant TB (MDR-TB) among new cases [1]. Culture-based drug susceptibility testing (DST) requires infrastructures often too sophisticated for resource-constrained settings. Detecting resistanceassociated mutations is a faster alternative, as shown by Genotype MTBDRplus (Hain Life science) [2] or Xpert MTB/RIF (Cepheid) [3]. To monitor drug resistance molecularly, the distribution of drug resistance-conferring

\footnotetext{
* Correspondence: Hans-Peter.Beck@unibas.ch

'Swiss Tropical and Public Health Institute, Socinstrasse 57, 4002 Basel, Switzerland

${ }^{2}$ University of Basel, Basel, Switzerland

Full list of author information is available at the end of the article
}

mutations in a given setting needs to be known, and such data is currently missing for PNG. We report mutations associated with drug resistance among $\mathrm{TB}$ isolates in the Madang area of PNG and provide evidence for transmission of drug-resistant $M$. tuberculosis.

\section{Results and discussion}

The patient characteristics and detailed M. tuberculosis genotypes were reported elsewhere [4]. In brief, 60 patients were recruited in the frame of a pilot study in 2005-2007 and 201 in the frame of a treatment cohort study in 2009-2010. History of previous TB treatment was reported in $16.9 \%(31 / 201)$ of the $2009-2010$ patients, for whom data was collected. Molecular analyses were performed on the DNA from 173 successfully grown isolates and phenotypic DST was obtained for 172 isolates. From the six previously described $M$.

\section{() Biomed Central}


tuberculosis lineages [5], we observed 133/173 (76.9\%) Euro-American (Lineage 4), 39/173 (22.5\%) East-Asian (Lineage 2, includes Beijing genotype), and 1/173 (0.6\%) Indo-Oceanic (Lineage 1).

Overall, $27 / 172(15.7 \%)$ isolates were resistant to $\geq 1$ drug: 15/172 (8.7\%) monoresistant, 3/172 (1.8\%) polyresistant and 9/172 (5.2\%) MDR. A total of 10/172 (5.8\%) strains were Rifampicin (RIF) resistant, 21/172 (12.2\%) Isoniazid (INH) resistant (13 low-level [0.1 $\mathrm{mg} / \mathrm{L}], 8$ high-level $[0.4 \mathrm{mg} / \mathrm{L}]), 9 / 172$ (5.2\%) Streptomycin (STR) resistant, and 4/172 (2.3\%) Ethionamide (ETH) resistant.

Among resistant isolates, the genes harboring drug resistance associated mutations were sequenced. The observed mutations in $k a t G$, inhA promoter, $a h p C$ promoter, $r p o B, e m b B, p n c A, r p s L, r r s$, gidB, and gyr $A$ are listed in Figure 1.

INH resistant isolates harbored mutations in katG (codon S315T) or inhA promoter (nucleotide C15T). All isolates with katG S315T were resistant to $0.4 \mathrm{mg} / \mathrm{L}$ INH except one, which was sensitive to this concentration of INH. On the other hand, all isolates with $\operatorname{inh} A$ promoter mutation were sensitive at this drug concentration (but resistant at $0.1 \mathrm{mg} / \mathrm{L}$ ), thus confirming the association between inhA promoter mutations and lowlevel INH resistance [6]. Among all 6/9 MDR-TB isolates with either $k a t G$ or $i n h A$ promoter mutations, all had

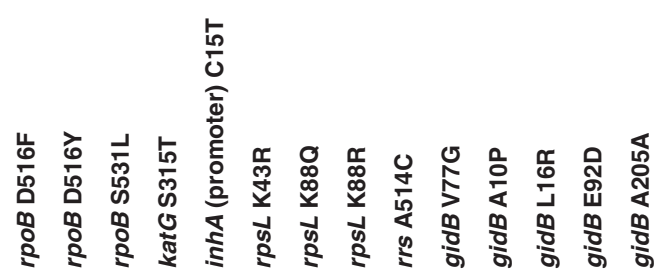

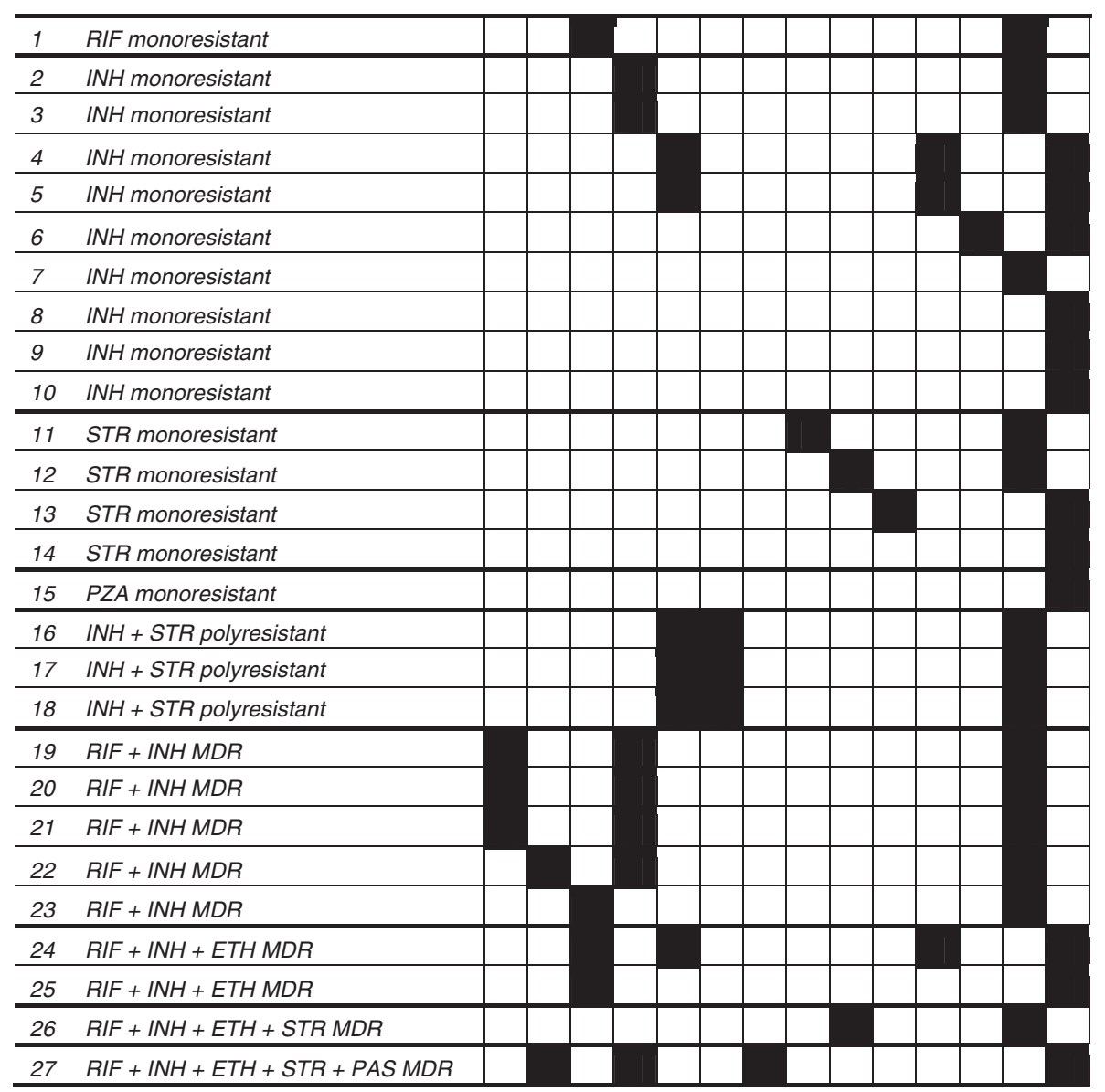

Figure 1 List of all mutations observed in each of the $\mathbf{2 7}$ strains resistant to at least one drug. The polymorphisms are indicated at codon positions, except for rrs gene. RIF: Rifampin; INH: Isoniazid; STR: Streptomycin; PZA: Pyrazinamide; ETH: Ethionamide; PAS: p-aminosalicylic acid; MDR: Multidrug resistant. 
the katG S315T mutation, except one with an inhA promoter mutation. This only MDR-TB case with an inhA promoter mutation belonged to the four MDR-TB cases, which were additionally ETH resistant. Mutations in inhA promoter have been shown to cause INH and ETH cross-resistance and were thereby associated with higher risks of extensively drug resistant TB [7].

Eight INH resistant strains (38.1\%) had no katG or inhA promoter mutation. Only 850 bp of katG were sequenced and mutations may therefore have been missed. However, katG mutations outside this region are rarer $[6,8,9]$. Alternatively, these strains might harbor mutation(s) in the $>20$ other genes reported as potentially associated with INH resistance (genes $i n i A$ or $x$ for example) [8].

We did not observe any $a h p C$ promoter mutation, thought to compensate the reduced catalase-peroxidase activity resulting from katG mutations [10,11]. Because the INH resistance-conferring mutations observed here, i.e. katG S315T and inhA promoter C15T, are known to be associated with a low fitness cost [11], they might not require compensation.

All RIF resistant isolates harbored mutations in rроB at codons D516F, D516Y or S531L except one, which did not have any mutation in the 600pb rpoB fragment sequenced. DST was repeated for this case, confirming the MDR phenotype. Furthermore, common rpoB, katG and inhA promoter mutations were excluded by Genotype MTBDRplus. Nevertheless, it has been estimated that mutations in the RIF resistance determining region (81-bp region in rpoB) account only for 95\% of RIF resistance [6] and therefore other mechanisms cannot be excluded. Mutation S531L has been linked to high-level RIF resistance [12], whereas D516Y was associated with low-level resistance [13-15]. Mutation D516F has only been reported in Kazakhstan [16] and may also cause low-level resistance. Low-level RIF resistance has been little considered, but could influence treatment, especially knowing that phenotypic DST outcomes may differ from the actual efficacy of the anti-TB drugs in patients [17].

STR resistant isolates harbored mutations in rpsL (codons K43R, K88Q, K88R) and rrs (nucleotide A514C), as previously reported $[18,19]$. One isolate was mutated at codon V77G in gidB, a mutation which was not reported before. One STR resistant isolate did not present any mutation in any of these genes.

Mutations in $\operatorname{gidB}$ have been associated with low-level STR resistance $[20,21]$, but were also reported in sensitive strains [22]. In this study, gidB mutations A10P, L16R, E92D, and A205A were observed among strains resistant to other drugs than STR. We further explored $\operatorname{gidB}$ mutations in whole genomes of 21 pan-susceptible strains representative of the six defined $M$. tuberculosis lineages [23]. Mutation gidB V77G, which we observed in one STR resistant isolate from PNG, could not be found in any of the 21 pan-susceptible strains. This mutation could therefore indeed be involved in drug resistance or could be a transitory polymorphism in the population. The mutation A10P observed in one STR sensitive isolate was not found in any of the 21 pansusceptible genomes. Mutations L16R was observed in genome sequences from Lineage 4 strains (Euro-American lineage) and E92D in Lineage 2 strains (East-Asian lineage). This supports the recent observation that gidB L16R occurred in LAM strains (i.e. Lineage 4), whereas gidB E92D occurred in Beijing strains [24]. A205A appeared mutated in all strains not belonging to Lineage 4 , therefore indicating that this mutation, identified by comparison to H37Rv, is a Lineage 4 mutation. Observations from the 21 pan-susceptible genomes suggest that most gidB mutations rather reflect $M$. tuberculosis lineage evolution than drug resistance.

Clusters were defined for strains sharing identical spoligotype and 24 locus mycobacterial interspersed repetitive unit variable number of tandem repeats (MIRUVNTR) patterns. Among isolates with complete patterns, 72/162 (44.4\%) were clustered. Despite potential fitness costs associated with resistance-conferring mutations [25], the proportion of clustered strains was not significantly different among drug-sensitive (60/137, 43.8\%) and drug-resistant $(12 / 25,48.0 \%)$ isolates of $M$. tuberculosis.

To distinguish between primary resistance and acquired resistance, clustered isolates sharing identical drug resistance-conferring mutations were considered. Five of the 12 (41.7\%) drug-resistant isolates involved in molecular clusters shared their drug resistanceconferring mutations with other isolates in the same cluster, thus strongly suggesting patient-to-patient transmission.

\section{Conclusions}

This study provides so far missing data about drug resistance-conferring mutations in $M$. tuberculosis isolates from Madang in PNG. Monitoring drug resistance is essential to prevent the spread of resistant bacteria, especially in diseases requiring lengthy treatments such as TB. Our data suggests that not all present drug resistance associated mutations may be detected by molecular tests, which mainly focus on a subset of polymorphisms only. However, given the complex implementation of culture-based DST in resource-constrained settings, PNG may be well suited for an accelerated roll-out of molecular drug resistance testing in order to better tackle the emergence and the transmission of drugresistant $M$. tuberculosis strains. 


\section{Methods}

\section{Study site and patient characteristics}

In 2005-2007, a pilot study was conducted in Madang (PNG) at the Modillion Hospital, which is the main point of care in Madang province. In April 2009, a cohort study was initiated in the same hospital and two smaller health centers in close vicinity to Madang town. Patients above 14 years were included if having microscopically confirmed pulmonary TB or other clinical evidence suggesting smear-negative TB. Treatment and follow-up were planed according to the directly observed treatment, short-course (DOTS) program. Demographic and clinical data were available for all patients, except those recruited during the 2005-2007 pilot study.

\section{Sample processing}

Sputum samples were examined by light microscopy after Ziehl-Neelsen staining. Decontamination was conducted according to Petroff's method [26]. DST was performed by proportion method [27] at the Queensland Mycobacterial Reference Laboratory in Australia using BACTEC $^{\mathrm{rm}}$ MGIT $^{\mathrm{m}} 960$ (Beckton Dickinson, USA) and the following drug concentrations: RIF $(1.0 \mu \mathrm{g} / \mathrm{mL}), \mathrm{INH}$ $(0.1$ and $0.4 \mu \mathrm{g} / \mathrm{mL})$, Ethambutol $(5.0 \mu \mathrm{g} / \mathrm{mL})$, Pyrazinamide $(100 \mu \mathrm{g} / \mathrm{mL})$, Streptomycin $(1.0 \mu \mathrm{g} / \mathrm{mL})$, Amikacin $(1.0 \mu \mathrm{g} / \mathrm{mL})$, Kanamycin $(5.0 \mu \mathrm{g} / \mathrm{mL})$, Ofloxacin $(2.0 \mu \mathrm{g} /$ $\mathrm{mL})$, Capreomycin $(2.5 \mu \mathrm{g} / \mathrm{mL})$, ETH $(5.0 \mu \mathrm{g} / \mathrm{mL}), p$ Aminosalicylic acid $(4.0 \mu \mathrm{g} / \mathrm{mL})$, and Cycloserine $(50.0 \mu \mathrm{g} / \mathrm{mL})$. Isolates resistant to one drug were categorized as monoresistant, isolates resistant to at least INH and RIF were categorized as MDR and isolates resistant to at least one drug but not MDR were considered polyresistant. DNA was extracted from cultures using Instigate Matrix (Bio-Rad, USA) and sent to the Swiss Tropical and Public Health Institute for molecular analyses.

\section{Strain genotyping}

Spoligotyping and 24 locus MIRU-VNTR were used to define strain clusters as previously described [28,29]. The online MIRU-VNTRplus platform was used for cluster identification (www.miru-vntrplus.org [30]). Clusters were defined for strains sharing identical spoligotype and 24 locus MIRU-VNTR patterns. Strains were assigned to one of the six previously described lineages by real-time PCR identification of specific single nucleotide polymorphisms (SNPs) [5,31-33].

\section{Drug resistance mutations}

The following genes (or gene regions) were sequenced to capture drug resistance conferring SNPs: rpoB, katG, inhA promoter, $a h p C$ promoter, embB, pncA, rpsL, rrs, $\operatorname{gid} B$, and $\operatorname{gyr} A$ (see Additional file 1: Table S1 for primers and PCR conditions). Sequencing was performed by Macrogen (The Netherlands). Observed mutations were compared to the online Tuberculosis Drug Resistance Mutation Database (TBDream, www.tbdreamdb. com [8]).

\section{Ethical approval}

The PNG Institute for Medical Research Review Board, and the PNG National Medical Research Advisory Council's Ethics Committee approved the study protocol. The Ethikkommission beider Basel in Switzerland was informed about the study. Written informed consent was obtained from all patients enrolled in the study.

\section{Additional file}

Additional file 1: Table 1 Primers and PCR conditions.

\section{Competing interests}

The authors declare that they have no competing interests.

\section{Authors' contributions}

MB carried out the molecular analyses, the data analyses and drafted the manuscript. PH conducted the patient recruitment and follow-up. SL participated to the study design. MC conducted the whole genome analyses. SN conducted the MIRU-VNTR analyses. RC conducted the phenotypic DST. CC participated in the phenotypic DST and helped to draft the manuscript. SB advised the molecular work and helped to draft the manuscript. PS contributed to the study set up. SP conceived the study design. SG participated in the design of the study, coordinated the molecular work and helped to draft the manuscript. Hans-Peter Beck participated in the design of the study, coordinated the molecular work and helped to draft the manuscript. All authors read and approved the final manuscript.

\section{Authors' information}

Co-senior author: Sebastien Gagneux and Hans-Peter Beck.

\section{Acknowledgments}

We thank all the study participants whose samples were used for analyses. We are indebted to the TB laboratory team in Madang.

This work was supported by the Swiss National Science Foundation (NorthSouth Program, grant number IZ70Z0_123988) and partially subsidized by a grant from the Stanley-Thomas Johnson Foundation and the Medicor Foundation, Lichtenstein

\section{Author details}

${ }^{1}$ Swiss Tropical and Public Health Institute, Socinstrasse 57, 4002 Basel, Switzerland. ${ }^{2}$ University of Basel, Basel, Switzerland. ${ }^{3}$ Papua New Guinea Institute of Medical Research, Goroka and Madang, Papua New Guinea. ${ }^{4}$ Borstel Research Center, Borstel, Germany. ${ }^{5}$ Queensland Mycobacterium Reference Laboratory, Pathology Queensland, Brisbane, Australia.

Received: 14 May 2012 Accepted: 6 August 2012

Published: 4 September 2012

\section{References}

1. World Health Organization: Tuberculosis country profile. Guinea: Papua New Guinea; 2011.

2. Hillemann D, Rüsch-Gerdes S, Richter E: Evaluation of the Genotype MTBDRplus assay for rifampin and isoniazid susceptibility testing of Mycobacterium tuberculosis strains and clinical specimens. J Clin Microbiol 2007, 45:2635-2640.

3. Boehme CC, Nicol MP, Nabeta P, Michael JS, Gotuzzo E, Tahirli R, Gler MT, Blakemore R, Worodria W, Gray C, Huang L, Caceres T, Mehdiyev R, Raymond L, Whitelaw A, Sagadevan K, Alexander H, Albert H, Cobelens F, Cox H, Alland D, Perkins MD: Feasibility, diagnostic accuracy, and 
effectiveness of decentralised use of the Xpert MTB/RIF test for diagnosis of tuberculosis and multidrug resistance: a multicentre implementation study. Lancet 2011, 377:1495-1505.

4. Ballif M, Harino P, Ley S, Carter R, Coulter C, Niemann S, Borrell S, Fenner L, Siba P, Phuanukoonnon S, Gagneux S, Beck H-P: Genetic diversity of Mycobacterium tuberculosis in Madang, Papua New Guinea. The international journal of tuberculosis and lung disease: the official journal of the International Union against Tuberculosis and Lung Disease 2012, 16:1100-1107.

5. Gagneux S, DeRiemer K, Van T, Kato-Maeda M, de Jong BC, Narayanan S, Nicol M, Niemann S, Kremer K, Gutierrez MC, Hilty M, Hopewell PC, Smal PM: Variable host-pathogen compatibility in Mycobacterium tuberculosis. Proc Natl Acad Sci U S A 2006, 103:2869-2873.

6. Zhang Y, Yew WW: Mechanisms of drug resistance in Mycobacterium tuberculosis [State of the art series. Drug-resistant tuberculosis. Edited by C-Y. Chiang. Number 1 in the series]. The International Journal of Tuberculosis and Lung Disease 2009, 13:1320-1330.

7. Müller B, Streicher EM, Hoek KGP, Tait M, Trollip A, Bosman ME, Coetzee GJ, Chabula-Nxiweni EM, Hoosain E: Gey van Pittius NC, Victor TC, van Helden $P D$, Warren RM: inhA promoter mutations: a gateway to extensively drug-resistant tuberculosis in South Africa? Int. J. Tuberc. Lung Dis 2011, 15:344-351.

8. Sandgren A, Strong M, Muthukrishnan P, Weiner BK, Church GM, Murray MB: Tuberculosis Drug Resistance Mutation Database. PLoS Med 2009, 6:e1000002.

9. Hazbón MH, Brimacombe M: Bobadilla del Valle M, Cavatore M, Guerrero MI, Varma-Basil M, Billman-Jacobe H, Lavender C, Fyfe J, García-García L, León Cl, Bose M, Chaves F, Murray M, Eisenach KD, Sifuentes-Osornio J, Cave MD, Ponce de León A, Alland D: Population Genetics Study of Isoniazid Resistance Mutations and Evolution of Multidrug-Resistant Mycobacterium tuberculosis. Antimicrob Agents Chemother 2006, 50:2640-2649.

10. Sherman DR, Mdluli K, Hickey MJ, Arain TM, Morris SL, Barry CE 3rd: Stover CK: Compensatory ahpC gene expression in isoniazid-resistant Mycobacterium tuberculosis. Science 1996, 272:1641-1643.

11. Gagneux S, Burgos MV, DeRiemer K, Enciso A, Muñoz S, Hopewell PC, Small PM, Pym AS: Impact of Bacterial Genetics on the Transmission of Isoniazid-Resistant Mycobacterium tuberculosis. PLoS Pathog 2006, 2:e61.

12. Telenti A, Imboden P, Marchesi F, Lowrie D, Cole S, Colston MJ, Matter L, Schopfer K, Bodmer T: Detection of rifampicin-resistance mutations in Mycobacterium tuberculosis. Lancet 1993, 341:647-650.

13. Van Deun A, Barrera L, Bastian I, Fattorini L, Hoffmann H, Kam KM, Rigouts L, Rüsch-Gerdes S, Wright A: Mycobacterium tuberculosis strains with highly discordant rifampin susceptibility test results. J Clin Microbiol 2009, 47:3501-3506.

14. van Ingen J, Aarnoutse R, de Vries G, Boeree MJ, van Soolingen D: Lowlevel rifampicin-resistant Mycobacterium tuberculosis strains raise a new therapeutic challenge. Int. J. Tuberc. Lung Dis 2011, 15:990-992.

15. Zaczek A, Brzostek A, Augustynowicz-Kopec E, Zwolska Z, Dziadek J: Genetic evaluation of relationship between mutations in rpoB and resistance of Mycobacterium tuberculosis to rifampin. BMC Microbiol 2009, 9:10.

16. Hillemann D, Kubica T, Agzamova R, Venera B, Rüsch-Gerdes S, Niemann S: Rifampicin and isoniazid resistance mutations in Mycobacterium tuberculosis strains isolated from patients in Kazakhstan. Int. J. Tuberc. Lung Dis 2005, 9:1161-1167.

17. Böttger $E C:$ The ins and outs of Mycobacterium tuberculosis drug susceptibility testing. Clin Microbiol Infect 2011, 17:1128-1134.

18. Sreevatsan S, Pan X, Stockbauer KE, Williams DL, Kreiswirth BN, Musser JM: Characterization of rpsL and rrs mutations in streptomycin-resistant Mycobacterium tuberculosis isolates from diverse geographic localities. Antimicrob Agents Chemother 1996, 40:1024-1026.

19. Honoré N, Cole ST: Streptomycin resistance in mycobacteria. Antimicrob Agents Chemother 1994, 38:238-242.

20. Okamoto S, Tamaru A, Nakajima C, Nishimura K, Tanaka Y, Tokuyama S, Suzuki Y, Ochi K: Loss of a conserved 7-methylguanosine modification in $16 \mathrm{~S}$ rRNA confers low-level streptomycin resistance in bacteria. Mol Microbiol 2007, 63:1096-1106.

21. Spies FS, da Silva PEA, Ribeiro MO, Rossetti ML, Zaha A: Identification of mutations related to streptomycin resistance in clinical isolates of
Mycobacterium tuberculosis and possible involvement of efflux mechanism. Antimicrob Agents Chemother 2008, 52:2947-2949.

22. Wong SY, Lee JS, Kwak HK, Via LE, Boshoff HIM, Barry CE: Mutations in gidB Confer Low-Level Streptomycin Resistance in Mycobacterium tuberculosis. Antimicrob Agents Chemother 2011, 55:2515-2522.

23. Comas I, Chakravartti J, Small PM, Galagan J, Niemann S, Kremer K, Ernst JD, Gagneux S: Human T cell epitopes of Mycobacterium tuberculosis are evolutionarily hyperconserved. Nat Genet 2010, 42:498-503.

24. Spies FS, Ribeiro AW, Ramos DF, Ribeiro MO, Martin A, Palomino JC, Rossetti MLR, da Silva PEA, Zaha A: Streptomycin Resistance and Lineage-Specific Polymorphisms in Mycobacterium tuberculosis gidB Gene. J Clin Microbiol 2011, 49:2625-2630

25. Borrell S, Gagneux S: Strain diversity, epistasis and the evolution of drug resistance in Mycobacterium tuberculosis. Clin Microbiol Infect 2011, 17:815-820.

26. Petroff SA: A New and Rapid Method for the Isolation and Cultivation of Tubercle Bacilli Directly from the Sputum and Feces. J Exp Med 1915, 21:38-42.

27. Canetti G, Fox W, Khomenko A, Mahler HT, Menon NK, Mitchison DA, Rist N, Smelev NA: Advances in techniques of testing mycobacterial drug sensitivity, and the use of sensitivity tests in tuberculosis control programmes. Bull. World Health Organ 1969, 41:21-43.

28. Kamerbeek J, Schouls L, Kolk A, van Agterveld M, van Soolingen D, Kuijper S, Bunschoten A, Molhuizen H, Shaw R, Goyal M, van Embden J: Simultaneous detection and strain differentiation of Mycobacterium tuberculosis for diagnosis and epidemiology. J Clin Microbiol 1997, 35:907-914

29. Supply P, Allix C, Lesjean S, Cardoso-Oelemann M, Rüsch-Gerdes S, Willery E, Savine E, de Haas $P$, van Deutekom $H$, Roring S, Bifani P, Kurepina N, Kreiswirth B, Sola C, Rastogi N, Vatin V, Gutierrez MC, Fauville M, Niemann S, Skuce R, Kremer K, Locht C, van Soolingen D: Proposal for standardization of optimized mycobacterial interspersed repetitive unit-variable-number tandem repeat typing of Mycobacterium tuberculosis. J Clin Microbiol 2006, 44:4498-4510.

30. Allix-Béguec C, Harmsen D, Weniger T, Supply P, Niemann S: Evaluation and strategy for use of MIRU-VNTRplus, a multifunctional database for online analysis of genotyping data and phylogenetic identification of Mycobacterium tuberculosis complex isolates. J Clin Microbiol 2008, 46:2692-2699

31. Hershberg R, Lipatov M, Small PM, Sheffer H, Niemann S, Homolka S, Roach JC, Kremer K, Petrov DA, Feldman MW, Gagneux S: High functional diversity in Mycobacterium tuberculosis driven by genetic drift and human demography. PLoS Biol 2008, 6:e311.

32. Comas I, Homolka S, Niemann S, Gagneux S: Genotyping of genetically monomorphic bacteria: DNA sequencing in Mycobacterium tuberculosis highlights the limitations of current methodologies. PLoS One 2009, 4:e7815.

33. Fenner L, Malla B, Ninet B, Dubuis O, Stucki D, Borrell S, Huna T, Bodmer T, Egger M, Gagneux S: "Pseudo-Beijing": Evidence for Convergent Evolution in the Direct Repeat Region of Mycobacterium tuberculosis. PLoS One 2011, 6:e24737.

doi:10.1186/1471-2180-12-191

Cite this article as: Ballif et al:: Drug resistance-conferring mutations in Mycobacterium tuberculosis from Madang, Papua New Guinea. BMC Microbiology 2012 12:191. 